\title{
Adaptive Tracking Control of SPROTT-H SYSTEM
}

\author{
Masoud Taleb Ziabari ${ }^{1}$ and Ali Reza Sahab ${ }^{2}$ \\ ${ }^{1}$ Faculty of Engineering, Computer Engineering Group, Ahrar University, Rasht, Iran. \\ ${ }^{2}$ Faculty of Engineering, Electrical Engineering Group, Islamic Azad University, Lahijan \\ Branch, Iran.
}

\begin{abstract}
This paper investigates the tracking problem of Sprott-H chaotic system which is three-dimensional chaotic systems discovered by Sprott (1994). New nonlinear control laws are derived for the tracking problem of uncertain Sprott-H chaotic system with unknown parameters. The adaptive generalized backstepping method is applied to control of uncertain Sprott-H chaotic system. Numerical simulations are presented to demonstrate the effectiveness of the control schemes.
\end{abstract}

\section{KEYWORDS}

Sprott-H Chaotic System, Uncertain, Adaptive Generalized Backstepping Method, Control.

\section{INTRODUCTION}

In recent years, various controllers have been proposed to achieve the control of chaotic systems [3-9]. The adaptive synchronization of an uncertain modified hyperchaotic Lü system was investigated in [10]. In [11], the output regulation problem for the Sprott-G chaotic system (1994) has been studied in detail. The stabilization and synchronization of the hyperchaotic Cai system with unknown system parameters was applied by adaptive control theory. The feedback controllers for control of the simplified Lorenz system was investigated in [13]. [14] derive state feedback controllers for the output regulation problem of the Sprott-H chaotic system (1994). In [15], active controller has been designed to solve the output regulation problem for the Sprott-K chaotic system (1994) and a complete solution for the tracking of constant reference signals (setpoint signals). [16] applied adaptive control theory for the control and synchronization of the Sprott H chaotic system (Sprott, 1994) with unknown system parameters.

The rest of the paper is organized as follows: In section 2, Sprott-H system is presented. In section 3, the generalized backstepping method is studied. In section 4, the generalized backstepping controller is designed for racking any desired inputs. In section 5, Represents simulation results. Finally, in section 6, Provides conclusion of this work. 


\section{SYSTEM DESCRIPTION}

The three-dimensional Sprott-H chaotic system [17] described by the dynamics

$$
\begin{aligned}
& \dot{x}=-a y+z^{2} \\
& \dot{y}=x+b y \\
& \dot{z}=x-z
\end{aligned}
$$

Where $a, b$ are positive constants and $x, y, z$ are variables of the system, when $a=1, b=0.5$, the system (1) is chaotic. See Figure 1 and Figure 2.

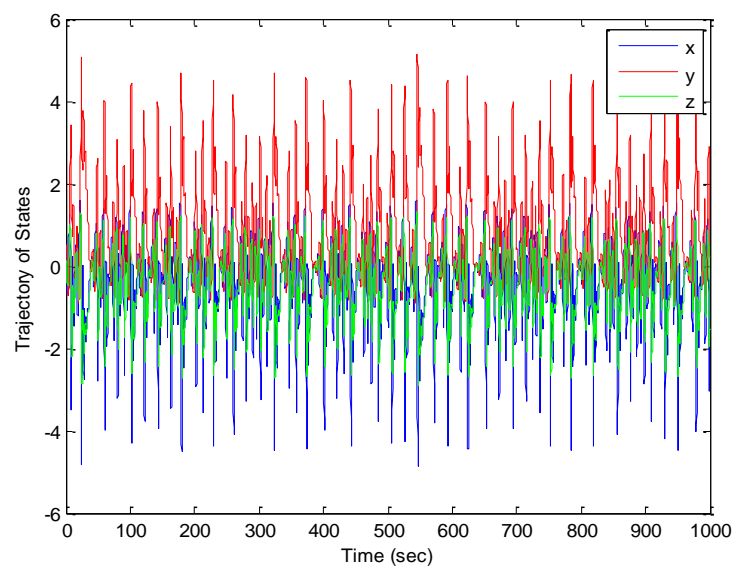

Figure 1. Time response of the system (1).

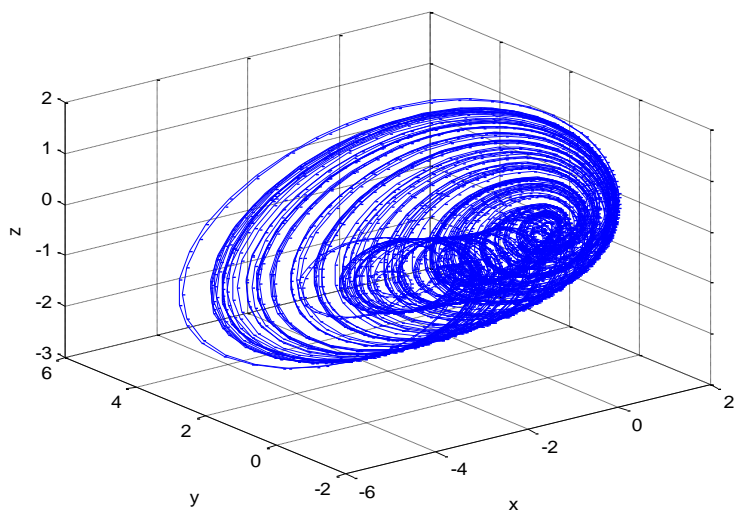

Figure 2. Phase portraits of the hyperchaotic attractors (1).

\section{Generalized BACKSTEPPING Method}

Generalized backstepping method [7-9] is applied to nonlinear systems as follow

$\left\{\begin{array}{l}\dot{X}=F(X)+G(X) \eta \\ \dot{\eta}=f_{0}(X, \eta)+g_{0}(X, \eta) u\end{array}\right.$

Where $\eta \in \Re$ and $x=\left[x_{1}, x_{2}, \cdots, x_{n}\right] \epsilon \Re$. Suppose the function $v(x)$ is the lyapunov function. 


$$
V(X)=\frac{1}{2} \sum_{i=1}^{n} x_{i}^{2}
$$

The control signal and the extended lyapunov function of system (2) are obtained by equations (4),(5).

$$
\begin{aligned}
& u=\frac{1}{g_{0}(X, \eta)}\left\{\begin{array}{c}
\sum_{i=1}^{n} \sum_{j=1}^{n} \frac{\partial \varphi_{i}}{\partial x_{j}}\left[f_{i}(X)+g_{i}(X) \eta\right] \\
-\sum_{i=1}^{n} x_{i} g_{i}(X)-\sum_{i=1}^{n} k_{i}\left[\eta-\varphi_{i}(X)\right]-f_{0}(X, \eta)
\end{array}\right\}, k_{i}>0, i=1,2, \cdots, n \\
& V_{t}(X, \eta)=\frac{1}{2} \sum_{i=1}^{n} x_{i}^{2}+\frac{1}{2} \sum_{i=1}^{n}\left[\eta-\varphi_{i}(X)\right]^{2}
\end{aligned}
$$

\section{Tracking of Chaotic System}

First, we will add control laws $u_{1}, u_{2}$ to the equation (1). let $\bar{z}$ be the deviation between the output $z$ and the desired trajectory $r(t), \bar{z}=z-r(t)$.

$$
\begin{aligned}
& \dot{x}=-a y+(\bar{z}+r)^{2}+u_{1} \\
& \dot{y}=x+b y+u_{2} \\
& \dot{\bar{z}}=x-\bar{z}-r-\dot{r}
\end{aligned}
$$

Stabilization of the state: In order to use the theorem, it is sufficient to establish equation (7). $\varphi_{1}(x, y, \bar{z})=r+\dot{r}$ $\varphi_{2}(x, y, \bar{z})=0$

According to the theorem, the control signals will be obtained from the equations (8).

$u_{1}=-(z-r)-k_{1}\left(x-\varphi_{1}\right)+\hat{a} y-z^{2}$

$u_{2}=-\left(\hat{b}+k_{2}\right) y-x$

The parameters $a, b$ are unknown and $\hat{a}, \hat{b}$ are respectively estimated values of parameters $a, b$ which are updated by following equation.

$\dot{\hat{a}}=-\hat{a} x y$

$\dot{\hat{b}}=\hat{b} y^{2}$

And Lyapunov function as

$V(x, y, z, w)=\frac{1}{2} x^{2}+\frac{1}{2} y^{2}+\frac{1}{2} \bar{z}^{2}+\frac{1}{2}\left(x-\varphi_{1}\right)^{2}+\frac{1}{2}\left(y-\varphi_{2}\right)^{2}$

we select the gains of controllers (8) in the following form

$k_{1}=10, k_{2}=9$

\section{NUMERICAL SIMULATION}

This section presents numerical simulations Sprott-H chaotic system. The generalized backstepping method (GBM) is used as an approach to control chaos in Sprott-H chaotic system. The initial values of the Sprott-H chaotic system are $x(0)=-1, y(0)=5, z(0)=-2$.

- $\quad$ Case 1 : Tracking $r(t)=1$, 
- Case 2 : Tracking $r(t)=\sin t$,

- Case 3: Tracking $r(t)=2-2 e^{-t}$.

Figure 3 shows that the scalar output can track the Step Input with the control inputs (8). Figure 4 shows that the scalar output can track the desired trajectory $r(t)=\sin (t)$. Figure 5 shows that the scalar output can track the desired trajectory $r(t)=2-2 e^{-t}$.

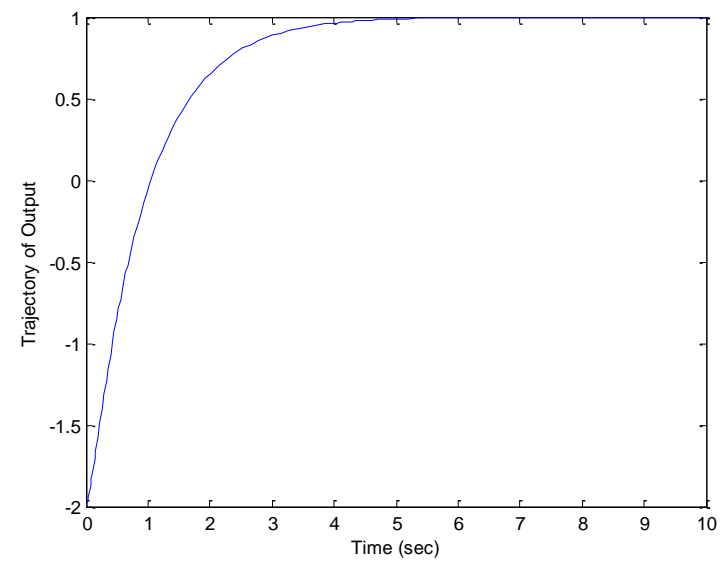

Figure 3. The time response of signal ( $z$ ) for tracks the Step Input.

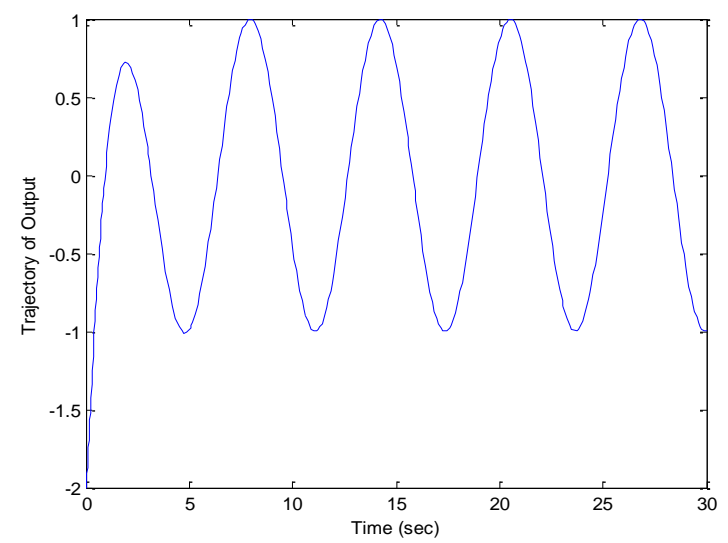

Figure 4. The time response of signal $(z)$ for tracks the desired trajectory $r(t)=\sin (t)$.

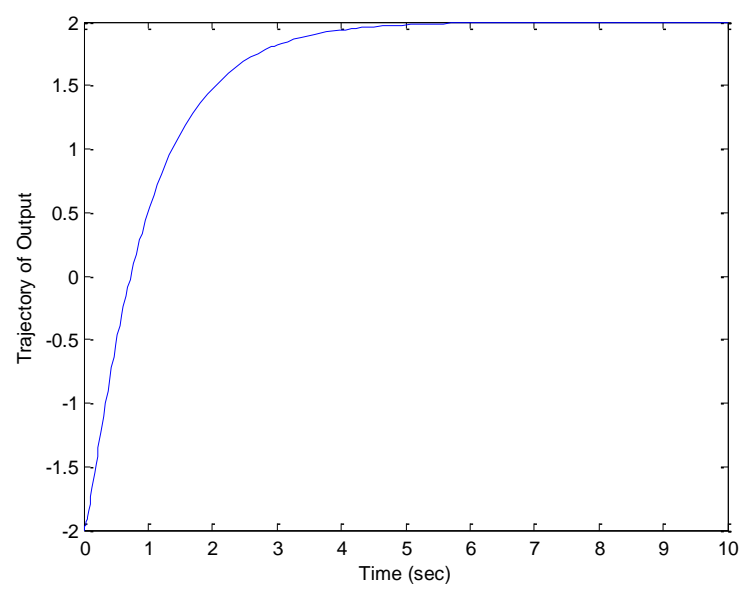

Figure 5. The time response of signal $(z)$ for tracks the desired trajectory $r(t)=2-2 e^{-t}$. 


\section{Conclusions}

In this paper, we applied adaptive generalized backstepping control for the control of the Sprott-H chaotic system with unknown system parameters. The tracking problem considered for the Sprott$\mathrm{H}$ chaotic system was for the tracking of any desired input signals. Numerical simulations show that the proposed method work effectively.

\section{REFERENCES}

[1] Chao-Chung Peng, Chieh-Li Chen. Robust chaotic control of Lorenz system by backstepping design. Chaos, Solitons and Fractals 37 (2008) 598-608.

[2] Cheng-Chi Wang, Neng-Sheng Pai, Her-Terng Yau. Chaos control in AFM system using sliding mode control by backstepping design. Commun Nonlinear Sci Numer Simulat 15 (2010) 741-751.

[3] Faqiang Wang, Chongxin Liu. A new criterion for chaos and hyperchaos synchronization using linear feedback control. Physics Letters A 360 (2006) 274-278.

[4] Yongguang Yu, Suochun Zhang. Adaptive backstepping synchronization of uncertain chaotic system. Chaos, Solitons and Fractals 21 (2004) 643-649.

[5] Sinha SC, Henrichs JT, Ravindra BA. A general approach in the design of active controllers for nonlinear systems exhibiting chaos. Int J Bifurcat Chaos 2000;10(1):165-78.

[6] M.T. Yassen. Chaos control of chaotic dynamical systems using backstepping design. Chaos, Solitons and Fractals 27 (2006) 537-548.

[7] Ali Reza Sahab and Mohammad Haddad Zarif. Improve Backstepping Method to GBM. World Applied Sciences Journal 6 (10): 1399-1403, 2009, ISSN 1818-4952.

[8] Sahab, A.R. and M. Haddad Zarif. Chaos Control in Nonlinear Systems Using the Generalized Backstopping Method. American J. of Engineering and Applied Sciences 1 (4): 378-383, 2008, ISSN 1941-7020.

[9] Ali Reza Sahab, Masoud Taleb Ziabari, Seyed Amin Sadjadi Alamdari. Chaos Control via Optimal Generalized Backstepping Method. International Review of Electrical Engineering (I.R.E.E), Vol.5, n.5.

[10] Sundarapandian Vaidyanathan, CONTROLLING HYPERCHAOS AND SYNCHRONIZATION OF AN UNCERTAIN MODIFIED HYPERCHAOTIC LÜ SYSTEM, International Journal of Instrumentation and Control Systems (IJICS) Vol.2, No.1, January 2012.

[11] Sundarapandian Vaidyanathan, OUTPUT REGULATION OF SPROTT-G CHAOTIC SYSTEM BY STATE FEEDBACK CONTROL, International Journal of Instrumentation and Control Systems (IJICS) Vol.1, No.1, July 2011.

[12] Sundarapandian Vaidyanathan, ADAPTIVE CONTROL AND SYNCHRONIZATION OF HYPERCHAOTIC CAI SYSTEM, International Journal of Control Theory and Computer Modelling (IJCTCM) Vol.1, No.1, July 2011.

[13] Sundarapandian Vaidyanathan, OUTPUT REGULATION OF THE SIMPLIFIED LORENZ CHAOTIC SYSTEM, International Journal of Control Theory and Computer Modelling (IJCTCM) Vol.1, No.3, November 2011.

[14] Sundarapandian Vaidyanathan, STATE FEEDBACK CONTROLLER DESIGN FOR THE OUTPUT REGULATION OF SPROTT-H SYSTEM, International Journal of Information Sciences and Techniques (IJIST) Vol.1, No.3, November 2011.

[15] Sundarapandian Vaidyanathan, ACTIVE CONTROLLER DESIGN FOR THE OUTPUT REGULATION OF SPROTT-K CHAOTIC SYSTEM, Computer Science \& Engineering: An International Journal (CSEIJ), Vol.2, No.3, June 2012.

[16] Dr. V. Sundarapandian, Adaptive Control and Synchronization of the Uncertain Sprott H System, International Journal of Advances in Science and Technology, Vol. 2, No.4, 2011.

[17] Sprott, J.C, Some simple chaotic flows, Phys. Rev. E., Vol. 50, pp 647-650. 